\section{Sighting dominance and utrocular discrimination}

\author{
CLARE PORAC \\ University of Victoria \\ Victoria, British Columbia, Canada \\ and \\ STANLEY COREN \\ University of British Columbia \\ Vancouver, British Columbia, Canada
}

Blake and Cormack $(1979 a, 1979 b)$ revived interest in utrocular discrimination, or the ability to detect the eyeof-origin of visual input, when they used it to investigate the properties of the neural network underlying binocular vision. In these and a later paper (Martens, Blake, Sloane, \& Cormack, 1981), they argued that detection of monocular origin is linked to the ocular dominance of cortical cells mediating binocular vision. They stated that the neural basis of binocular vision resides in an interacting pool of neurons that receive input from both eyes. However, individual neurons differ in their ability to be driven by either the right or left eye; in other words, they display ocular dominance. Observers can detect ocular origin reliably if these interacting groups of binocular neurons show a combined skewed response favoring one eye or the other.

This model can account for the individual differences in utrocular discrimination ability reported in several studies (see Blake \& Cormack, 1979b; Enoch, Goldman, \& Sunga, 1969). Individuals such as the stereoblind possess binocular neural networking that is strongly asymmetrical (driven primarily by one eye). As evidence for the model, these individuals should show good utrocular discrimination performance, a finding that has been reported experimentally (Blake \& Cormack, 1979a). However, other observers, who do not have detectable abnormalities of binocular vision, also show utrocular discrimination ability. Individual differences in the degree of asymmetry of binocular neural activity, of a less extreme form than that presumed to exist in stereoblind observers, could account for these individual differences in discrimination performance.

Recently, Porac and Coren (1984) argued that strong sighting dominance, the consistent use of the same eye during binocular alignment tasks, is another individual difference characteristic that may predict good utrocular

\footnotetext{
This research was supported by grants from the Natural Sciences and Engineering Research Council of Canada and represents an equal and shared contribution of both authors. We thank P. Balazi, C. BehmanSummerfeldt, K. Cooper, and L. McRae for their assistance in the collection of these data and L. M. Ward for advice on data analysis procedures. Address reprint requests to C. Porac, Department of Psychology, University of Victoria, Victoria, British Columbia, Canada V8W $2 \mathrm{Y} 2$.
}

discrimination ability. However, despite the presence of strong sighting dominance behaviors in all of their observers, Porac and Coren (1984) obtained reliable utrocular discrimination only when they provided feedback about performance accuracy. Discrimination between sightingand nonsighting-eye input was at chance levels in the absence of feedback. In a final study in this series, which did not involve utrocular discrimination, observers judged sighting-eye input to have a different image quality from that of nonsighting-eye input. Therefore, Porac and Coren (1984) proposed that feedback during utrocular discrimination called attention to these image differences, which then were used to perform the eye-of-origin discrimination.

Although the Porac and Coren (1984) study implicates sighting dominance as a factor in utrocular discrimination, it does not permit an analysis in the framework of the Blake and Cormack (1979b) model. The overt behavior of consistent sighting dominance may indicate an underlying asymmetrical pattern of neural activity, similar to, but less extreme than, that proposed to exist in stereoblind observers. Detectable image quality differences between the sighting and nonsighting eyes could be phenomenal byproducts of this neural asymmetry. Therefore, observers with strong sighting dominance should display good utrocular discrimination ability in the absence of feedback about performance, given sufficient exposure to the utrocular task. We conducted the present study to examine the role of sighting dominance in utrocular discrimination in the context of the Blake and Cormack (1979b) model of the task.

\section{Utrocular Paradigm and Results}

Eight observers with excellent visual acuity in each eye and normal binocular functions were tested. We assessed their sighting dominance with repeated trials of the Point Test and the Miles ABC Test (see Porac \& Coren, 1981). All participants showed strong sighting dominance characteristics, defined as the consistent use of one eye for sighting on at least $75 \%$ of the sighting trials. We selected 4 right-sighters and 4 left-sighters for the final sample ( 7 males and 1 female).

The stimuli and apparatus were identical to those of Porac and Coren (1984), who described them extensively in that paper. Briefly, we presented the experimental targets in an Iconix four-channel tachistoscope. Orthogonally oriented polarizers in the channels and analyzers in the viewing ports separated the two monocular fields. During the preexposure interval, observers viewed a sniall binocular fixation cross. During monocular testing, an outline white square was presented for $250 \mathrm{msec}$. This square surrounded the fixation cross in the stimulated eye while the unexposed eye viewed a uniform black field. We presented the square and fixation cross to both eyes on binocular trials. The postexposure field was, once 
again, the binocular fixation cross. Phenomenally, the stimulus display appeared to be a brief enclosure of the fixation cross by the white square.

Each observer received a random sequence of 96 presentations at each session. They included 32 trials of right eye, left eye, and binocular exposures. The experimental task was a three-alternative forced choice. The observers fixated the white cross in the preexposure field. When a ready signal was given, they were told that the white square would surround the white cross briefly, and that they were to respond whether the square was presented to the right, left, or both eyes. We did not permit responses of "don't know." Each observer received five sessions of 96 trials each for a total of 480 trials; these sessions were conducted on 5 consecutive days.

We tabulated the data separately for the sighting, nonsighting, and binocular exposure conditions. We defined the probability of a correct response $P$ (hit) as the number of correct choices divided by the total number of presentations for an exposure condition. The mean $P($ hit $)$ for all observers is plotted against day of testing for each exposure condition in Figure 1A. Observers identified binocular stimuli with greater accuracy than they did monocular exposures. The exposure effect was statistically significant $[F(2,14)=9.56, p<.01]$. Observers could discriminate binocular from monocular stimulation; however, they could not discriminate between monocular inputs.

Since we used a three-alternative forced-choice procedure, we conducted a series of $t$ tests to compare the obtained performance for each exposure condition with a theoretical chance level of responding, or a $P($ hit) of 0.33 . Neither of the two monocular conditions differed significantly from chance on any day of testing. This poor monocular performance can be compared with that in the binocular condition, where the detection of binocular stimulation was significantly greater than chance levels $(p<.05)$ for each of the 5 days.

In the Porac and Coren (1984) study, observers originally showed no utrocular discrimination; however, in the presence of error feedback, they soon developed reliable eye-of-origin discrimination. In the present study, we observed no systematic practice effect $[F(4,28)=1.64]$, despite the large number of trials over an extended time period. Simple experience with the utrocular discrimination task was not sufficient to establish an ability to discriminate between the monocular inputs.

We reanalyzed the data with signal detection procedures to assess the effect of response bias factors. Since we had computed the $P($ hit) for each exposure for every observer, we also computed the $P$ (false alarm), which, together with $P($ hit), allowed us to convert the data into sensitivity and response bias measures. We tallied false alarms by counting the number of confusions for each exposure (that is, the number of "right" responses to left eye or binocular stimulation); we then divided this value by the total number of presentations in the other two conditions. We used the $P($ hit) and $P$ (false alarm) values for every observer
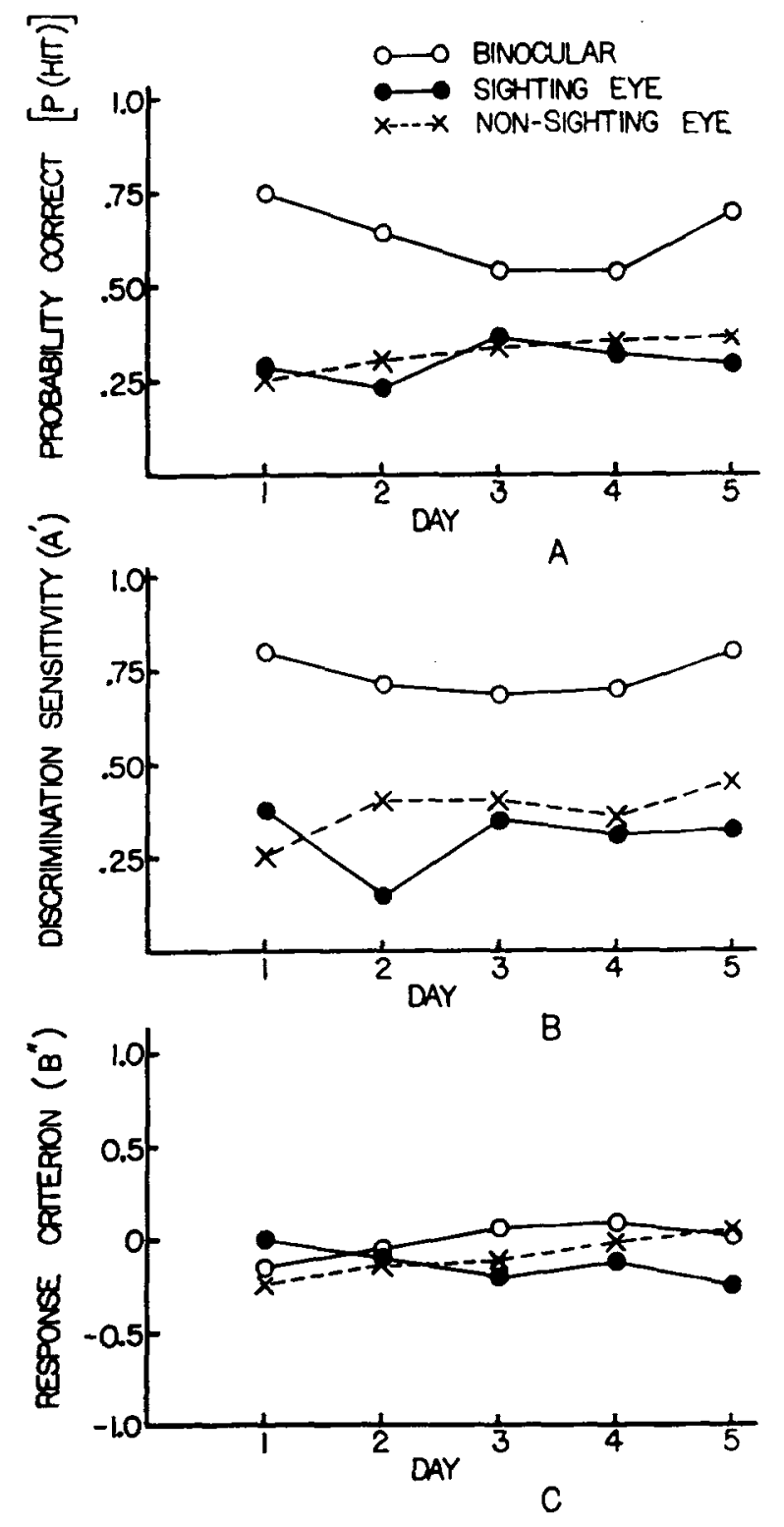

Figure 1. (A) Mean $P($ hit) for binocular, sighting-eye, and nonsighting-eye exposures plotted against day of testing. (B) $A^{\prime}$ scores showing no increase in sensitivity for any exposure condition over 5 days of testing. (C) B" scores showing no change in response criterion over days of testing.

to compute values of $A^{\prime}$ and $B^{\prime \prime}$, nonparametric measures of sensitivity, and response criterion, respectively (see Grier, 1971, and Porac \& Coren, 1984). The $A^{\prime}$ index varies between 0 and 1.0 , with a value of .5 representing the chance level of responding; $B^{\prime \prime}$ ranges from -1.0 to +1.0 , with negative numbers indicating a lax response criterion and positive numbers, a strict criterion.

Figures $1 \mathrm{~B}$ and $1 \mathrm{C}$ indicate that the mean $A^{\prime}$ results mimic those found when we analyzed the data as $P($ hit $)$. Observers recognized binocular stimulation, but they did not discriminate between the two monocular views, result- 
ing in a significant main effect of exposure condition $[F(2,14)=9.36, p<.01]$. Individual $t$ tests showed that the $A^{\prime}$ values for the binocular condition were significantly greater than chance, whereas the comparisons of the monocular performance scores never reached statistical significance. Also, there was no systematic practice effect. Figure $1 \mathrm{C}$ shows no apparent differences in response criteria across the three exposure conditions. This interpretation is supported by the absence of a main effect for exposure condition in the analysis of the $B^{\prime \prime}$ scores. Thus, response bias was not a factor in the obtained pattern of results. The ability to discriminate binocular from monocular input and the lack of response bias in the utrocular task are findings that replicate those of Porac and Coren (1984).

\section{Conclusion}

The present results suggest two interpretations. First, if utrocular discrimination depends on monocularly skewed activity patterns in pools of binocular neurons, as proposed by the Blake and Cormack (1979b) model, our findings suggest that behavioral manifestations of strong sighting dominance are not maintained by such neural asymmetries. If neural asymmetry is an underlying factor in the development of strong sighting dominance, our observers should have been able to make monocular discriminations above chance levels, at least toward the end of the 5-day testing interval. Blake and his colleagues found that accurate utrocular discrimination depended on the spatial frequency components of the stimulus array (Blake \& Cormack, 1979a). Perhaps the current experimental stimuli did not contain the appropriate frequency range to evoke accurate monocular discrimination performance. However, a more likely interpretation, given the length of the testing period, is that strong sighting dominance, in and of itself, cannot predict the likelihood of accurate utrocular discrimination.

Second, the present findings support the interpretation of a "weak" form of eye signature, a notion suggested in the Porac and Coren (1984) report. Eye-of-origin information signaled by the skewed activity pattern of binocular neurons is a "strong" form of eye signature in that the coding of eye-of-origin is contained in the neural network maintaining monocular combination. However, observers may discriminate right- from lefteye input on the basis of image quality differences caused by asymmetries of peripheral mechanisms in the visual system (Ono \& Barbeito, 1985; Steinbach, Howard, \& Ono, 1985). For example, differences in ocular structure in one eye could affect light transmission in that eye. Although traditional assessments of visual abilities may not detect these subtle differences, observers may use this information to make utrocular discriminations under the appropriate circumstances. In light of the present results, it seems likely that feedback in the utrocular situation calls attention to these subtle ocular differences, allowing chance levels of monocular discrimination to increase to above chance performance. In the absence of corrective feedback, observers do not attend to the differences and utrocular discrimination remains poor. It is likely, then, that reported monocular image quality differences between the sighting and the nonsighting eyes are based on these eye-signature processes and not on neural asymmetries at more central levels of the visual system (Porac \& Coren, 1981, 1984). This viewpoint agrees with that of Barbeito, Levi, Klein, Loshin, and Ono (1985), who failed to replicate Blake and Cormack (1979a) and did not find utrocular discrimination ability in stereoblind observers. To explain these results, they proposed that the effects of asymmetrical neural processing might not be available to the conscious percept. In other words, the activity of skewed distributions of neurons need not result in noticeable differences in the phenomenal experience of the two eyes' views. These circumstances predict an independence between the report of phenomenal differences between the eyes and utrocular performance studied under conditions in which all peripheral differences have been eliminated.

\section{REFERENCES}

Barbeito, R., Levi, D., Klein, S., Loshin, D., \& ONo, H. (1985). Stereo-deficients and stereoblinds cannot make utrocular discriminations. Vision Research, 25, 1345-1348.

Blake, R., \& CoRmaCK, R. (1979a). Psychophysical evidence for a monocular visual cortex in stereoblind humans. Science, 203, 274-275.

Blake, R., \& CoRmack, R. (1979b). Utrocular discrimination. Perception \& Psychophysics, 26, 53-68.

Enoch, J., Goldman, H., \&unga, T. (1969). The ability to distinguish which eye was stimulated by light. Investigative Ophthalmology, 8, 317-331.

GrIER, J. B. (1971). Nonparametric indexes for sensitivity and bias: Computing formulas. Psychological Bulletin, 75, 424-429.

Martens, W., Blake, R., Sloane, M., Cormack, R. H. (1981). What masks utrocular discrimination. Perception \& Psychophysics, 30, 521-532.

ONO, H., \& BARBEITo, R. (1985). Utrocular discrimination is not sufficient for utrocular identification. Vision Research, 25, 289-299.

PORAC, C., \& COREN, S. (1981). Lateral preferences and human behavior. New York: Springer.

PORAC, C., \& COREN, S. (1984). Monocular asymmetries in vision: A phenomenal basis for eye signature. Canadian Journal of Psychology, 38, 610-624.

Steinbach, M. J., Howard, I. P., \& ONo, H. (1985). Monocular asymmetries in vision: We don't see eye-to-eye. Canadian Journal of Psychology, 39, 476-478.

(Manuscript received March 14, 1986; accepted for publication April 17, 1986.) 